\title{
A Left-First Search Algorithm for Planar Graphs*
}

\author{
H. de Fraysseix, ${ }^{1}$ P. O. de Mendez, ${ }^{1}$ and J. Pach ${ }^{2}$ \\ ${ }^{1}$ Centre de Mathématiques Sociales, EHESS, \\ 54 Boulevard Raspail, 75006 Paris, France \\ hf@alize.msh-paris.fr \\ ${ }^{2}$ Department of Computer Science, City College, CUNY, \\ New York, NY 10031, USA \\ and \\ Courant Institute, N.Y.U., 251 Mercer Street, \\ New York, NY 10012, USA \\ pach@cims6.nyu.edu
}

\begin{abstract}
We give an $O(|V(G)|)$-time algorithm to assign vertical and horizontal segments to the vertices of any bipartite plane graph $G$ so that (i) no two segments have an interior point in common, and (ii) two segments touch each other if and only if the corresponding vertices are adjacent. As a corollary, we obtain a strengthening of the following theorem of Ringel and Petrovič. The edges of any maximal bipartite plane graph $G$ with outer face $b w b^{\prime} w^{\prime}$ can be colored by two colors such that the color classes form spanning trees of $G-b$ and $G-b^{\prime}$, respectively. Furthermore, such a coloring can be found in linear time. Our method is based on a new linear-time algorithm for constructing bipolar orientations of 2-connected plane graphs.
\end{abstract}

\section{Introduction}

Throughout this paper we consider only finite graphs $G$ without loops, but we allow multiple edges. If $G$ has no multiple edges, then it is called a simple graph. A graph is 2-connected if it cannot be disconnected by the removal of a vertex.

Let $\vec{G}$ be a directed graph obtained by orienting the edges of $G$. A vertex of $\vec{G}$ is said to be a source (sink) if its indegree (outdegree) is $0 . \vec{G}$ is acyclic if it contains no

* The research of $H$. de Fraysseix and P. O. de Mendez was supported by ESPRIT Basic Research Action No. 7141 (ALCOM II). J. Pach's research was supported by NSF Grant CCR-9122103, OTKA-4269, and ALCOM II. 
oriented cycle. For any partition of the vertex set $V(\vec{G})=V_{1} \cup V_{2}$, the family of edges between $V_{1}$ and $V_{2}$ is said to form a cocycle (or an oriented cut) if all of them are oriented toward $V_{2}$.

The following concept was introduced by Lempel et al. [LEC] to design an efficient planarity-testing algorithm. It plays a crucial role in many problems about graph drawings, motion planning, visibility, and incidence relations between geometric objects, etc. [EET], [FMR], [FPP], [FRU], [OW], [P2], [R2], [R3], [RT], [T1], [T2], [TT1], [TT2].

Definition 1.1. Given an edge $\vec{e}=\overrightarrow{s t}$ of $\vec{G}$, we say that the orientation of $\vec{G}$ is $\vec{e}$-bipolar (or defines an st-ordering) if:

(a) $\vec{G}$ is acyclic, and

(b) $s$ and $t$ are the unique source and sink of $\vec{G}$, respectively.

We also use another equivalent form of this definition (which can easily be extended to matroids).

Lemma 1.2. Given an edge $\vec{e}$ of $\vec{G}$, the orientation of $\vec{G}$ is $\vec{e}$-bipolar if and only if:

(a') every edge of $\vec{G}$ belongs to a cocycle, and

(b') every cocycle of $\vec{G}$ contains $\vec{e}$.

Proof. Obviously, (a') implies (a). Conversely, if $\vec{G}$ is acyclic and $\vec{e}^{\prime}=\overrightarrow{s^{\prime} t^{\prime}}$ is any edge, then let $V_{2}$ be the set of all vertices that can be reached from $t^{\prime}$ by a directed path, and let $V_{1}=V(\vec{G})-V_{2}$. Then all edges between $V_{1}$ and $V_{2}$, including $\vec{e}^{\prime}$, are oriented toward $V_{2}$, thus ( $\left.\mathrm{a}^{\prime}\right)$ holds.

To show that (b) implies ( $\left.\mathrm{b}^{\prime}\right)$ for any acyclic digraph $\vec{G}$, it is enough to observe that, if a partition $V(\vec{G})=V_{1} \cup V_{2}$ defines a cocycle, then $V_{1}$ and $V_{2}$ must contain a source and a sink, respectively. Thus, $s \in V_{1}, t \in V_{2}$, and $\vec{e}=\overrightarrow{s t}$ belongs to this cocycle. Conversely, if an acyclic digraph satisfies $\left(\mathrm{b}^{\prime}\right)$ with $\vec{e}=\overrightarrow{s t}$ then, for any source $x$ (and sink $y$ ), the collection of edges incident to $x(y)$ forms a cocycle. Consequently, $\vec{e}$ is incident to both $x$ and $y$. Hence, $x=s, y=t$, and (b) holds.

Corollary 1.3. If $\vec{G}$ has an $\vec{e}$-bipolar orientation, then it has no two cocycles such that one contains the other.

Proof. Let $E$ and $E^{\prime}$ be two cocycles of $\vec{G}$ defined by the partitions $V_{1} \cup V_{2}$ and $V_{1}^{\prime} \cup V_{2}^{\prime}$, respectively, where $s \in V_{1} \cap V_{1}^{\prime}$ and $t \in V_{2} \cap V_{2}^{\prime}$. Suppose without loss of generality that $W_{1}=V_{1}^{\prime} \cap V_{2} \neq \varnothing$. If $E \subseteq E^{\prime}$, then $W_{1}$ and $W_{2}=V(G)-W_{1}$ define a cocycle which does not contain $\vec{e}=\overrightarrow{s t}$, contradicting condition $\left(b^{\prime}\right)$ in Lemma 1.2 .

If $\vec{G}$ has an $\vec{e}$-bipolar orientation, then its underlying graph $G$ (obtained by disregarding the orientation of the edges) is obviously 2 -connected. Indeed, if $G$ fell 
into two components $G_{1}$ and $G_{2}$ by the removal of a vertex $x$, then, by the acyclicity of $\vec{G}$, both parts of $\vec{G}$ induced by $V\left(G_{1}\right) \cup\{x\}$ and $V\left(G_{2}\right) \cup\{x\}$ would contain a source and a sink, contradicting condition (b) of Definition 1.1.

On the other hand, it is easy to see that, given any 2-connected graph $G$ and any edge $e=s t$, an $\vec{e}$-bipolar orientation of the edges of $G$ exists with $\vec{e}=\overrightarrow{s t}$. Moreover, Even and Tarjan [ET] devised a linear-time algorithm to find such an orientation, which has been subsequently simplified by Ebert [E] and Tarjan [T2].

In Section 2 of this paper we propose an equally fast but much simpler greedy algorithm based on Whitney's theorem [W] to find bipolar orientations of 2connected plane graphs.

A plane graph is a planar graph embedded in the plane (or in the sphere) so that its edges are represented by simple noncrossing Jordan arcs. If a plane graph $G$ with at least three vertices is 2-connected, then its dual graph $G^{*}$ can be defined as follows. Put a vertex of $G^{*}$ in each face of $G$ and, if two faces meet along an edge $f$, then connect the corresponding two vertices by an arc $f^{*}$ crossing $f$. (It is well known and easy to see that this construction can be carried out so that we obtain a plane graph $G^{*}$. By the 2-connectedness of $G, G^{*}$ has no loops, but it may have multiple edges.) Any orientation of $G$ induces a dual onientation of $G^{*}$ in a natural way: we obtain the orientation of $f^{*}$ from that of $f$ by a clockwise turn.

Theorem 1.4. Let $\vec{G}$ be an $\vec{e}$-bipolar orientation of a 2-connected plane graph with at least three vertices, and let $\vec{G}^{*}$ denote its dual graph with the dual orientation. Then the directed graph $\vec{G}_{-}^{*}$ obtained from $\vec{G}^{*}$ by reversing the orientation of $\vec{e}^{*}$ is $\vec{e}_{-}^{*}$-bipolar oriented, where $\vec{e}_{-}^{*}$ and $\vec{e}^{*}$ are opposite orientations of the same edge.

Proof. We show that $\vec{G}_{-}^{*}$ satisfies conditions (a') and (b) of Lemma 1.2 and Definition 1.1, respectively.

Let $\vec{G}_{-}$denote the digraph obtained from $\vec{G}$ by changing the orientation of $\vec{e}=$ $\overrightarrow{s t}$ to $\vec{e}_{-}=\overrightarrow{s t}$. Any edge of $\vec{G}$ can be extended to a directed path in $\vec{G}$ connecting $s$ to $t$. Thus, any edge $\vec{f} \in E\left(\vec{G}_{-}\right)$belongs to a (simple) cycle of $\vec{G}_{-}$passing through $\vec{e}_{-}$. The edges of $\vec{G}_{-}^{*}$ crossing this cycle form a cocycle containing $\vec{f}^{*}$ (and $\vec{e}_{-}^{*}$ ), which proves $\left(\mathrm{a}^{\prime}\right)$.

Suppose, for contradiction, that $\vec{G}_{-}^{*}$ does not satisfy (b). Let $s^{*}$ and $t^{*}$ denote the endpoints of $\vec{e}_{-}^{*}\left(\vec{e}_{-}^{*}=\vec{s}^{*} t^{*}\right)$, and assume without loss of generality that $\vec{G}_{-}^{*}$ has a source $x$ different from $s^{*}$. Clearly, $x \neq t^{*}$. Those edges of $\vec{G}_{-}$which cross an edge incident to $x$ form a cycle. Since this cycle does not use the arc $e$, this would also be a cycle in $\vec{G}$, a contradiction.

As any graph which has a bipolar orientation is 2-connected and vice versa, Theorem 1.4 immediately implies that the dual of a 2-connected plane graph is also 2-connected.

In Section 3 we apply the above concepts and results to obtain the following theorem. 
Theorem 1.5. A linear-time algorithm exists which assigns vertical and horizontal segments to the vertices of any bipartite plane graph $G$ so that:

(i) No two segments have an interior point in common.

(ii) Two segments touch each other if and only if the corresponding vertices are adjacent in $G$.

Note that if the black and white vertices of a bipartite (2-colored) graph $G$ can be represented by vertical and horizontal segments, respectively, satisfying conditions (i) and (ii), then $G$ is necessarily planar.

We say that a graph $G$ has a segment representation if its vertices can be represented by segments in the plane so that two segments cross each other if and only if the corresponding vertices are adjacent. It was shown in [HNZ] that any bipartite planar graph can be represented in such a way. Note that this fact is an immediate corollary to Theorem 1.5. However, it is not known whether every planar graph admits a segment representation.

Definition 1.6. A bipartite plane graph is called a quadrilateralization if it contains no multiple edges and each of its faces has four edges.

It is easy to see that every quadrilateralization is 2-connected.

Given a bipartite plane graph, in linear time we can remove all multiple edges (by lexicographically bucket-sorting all edges with respect to their endpoints). Then we can use any naive linear-time algorithm to extend the remaining graph to a quadrilateralization, by adding edges and vertices. Thus, it is sufficient to prove Theorem 1.5 for quadrilateralizations.

Definition 1.7 [R1]. Let $H$ be a connected plane graph. Triangulate every face $f$ of $H$ from one of its interior points $x_{f}$ (by connecting $x_{f}$ to the vertices of $f$ ), and delete all edges belonging to $H$. The resulting graph $A(H)$ is called the angle graph of $H$.

Remark 1.8. Let $H$ be a connected plane graph. Then $A(H)$ is a quadrilateralization if and only if $H$ is 2-connected.

On the other hand, every quadrilateralization can be obtained as the angle graph of some 2-connected plane graph.

Lemma 1.9. Let $G$ be a quadrilateralization whose vertices are colored black and white. For every face $f$ of $G$, connect its two black (white) vertices by an edge within $f$. The graph $G_{\mathrm{b}}\left(G_{\mathrm{w}}\right)$ formed by these edges is called the graph of black (white) diagonals of $G$. Then

$$
A\left(G_{\mathrm{b}}\right)=A\left(G_{\mathrm{w}}\right)=G \text {. }
$$

Corollary 1.10. $G_{\mathrm{b}}$ and $G_{\mathrm{w}}$ are 2-connected plane graphs, dual to each other.

Proof. Immediately follows from Remark 1.8.

For some related results, see [DLR] and [TT3]. 
In Section 4 of this paper we apply our technique to give a simple alternative proof of a theorem of Ringel [R1] and Petrovič [P1] on quadrilateralizations (see Corollary 4.1).

\section{Greedy Algorithm for Bipolar Orientation}

\subsection{The General Scheme}

Let $G$ be any 2-connected graph with $n$ vertices and $m$ edges. For any edge $s t$ of $G$, $G$ has a Whitney decomposition into handles, i.e., there is a nested sequence of subgraphs $G_{0}=\{s t\} \subset G_{1} \subset G_{2} \subset \cdots \subset G_{k}=G$ such that $G_{i+1}$ can be obtained from $G_{i}$ by the addition of a simple path $P_{i+1}$ which has only its endpoints in common with $G_{i}$.

First we present a simple general algorithm which maintains a total ordering of the vertices of $G_{i}$ such that every $P_{j}(j \leq i)$ forms a monotone chain. Directing every edge of $G$ toward its larger endpoint in the final ordering, we obtain an st-ordering (bipolar orientation) of $G$. In fact, our algorithm will also maintain the orientation of the edges of $G_{i}$ compatible with the ordering of its vertices.

Suppose that we have already found a sequence of subgraphs $G_{j}(j \leq i)$ with the above properties, and that the vertices of $G_{i}$ are totally ordered by a linked list called "LINK." Assume further that all edges of $G_{i}$ are oriented toward their higher endpoints in this order, and every $P_{j}(j \leq i)$ forms an oriented path. A vertex $x \in V\left(G_{i}\right)$ is said to be saturated, if all edges of $G$ incident to $x$ belong to $G_{i}$. Step $i$ $(i \geq 0)$ of our algorithm consists of three parts:

(1) Find the first unsaturated vertex $x \in V\left(G_{i}\right)$ on the list LINK.

(2) Find a simple path $P_{i+1}$ in $G-G_{i}$ connecting $x$ to some other vertex $y \in V\left(G_{i}\right)$ such that no internal point of $P_{i+1}$ belongs to $V\left(G_{i}\right)$. Orient the edges of $P_{i+1}$ from $x$ toward $y$.

(3) Insert the internal vertices of $P_{i+1}$ in the list LINK between $x$ and LINK $[x]$, i.e., immediately after $x$.

If we cannot execute (1), i.e., all vertices of $G_{i}$ are saturated, then $G_{i}=G$ and our algorithm ends. Otherwise, let $x^{\prime}$ be any neighbor of $x$ such that the edge $x x^{\prime}$ does not belong to $G_{i}$. If $x^{\prime} \in V\left(G_{i}\right)$, then $x^{\prime}$ is also unsaturated, so $x$ precedes $x^{\prime}$ on the list LINK. In this case, $P_{i+1}$ consists of the single edge $x x^{\prime}$ oriented from $x$ to $x^{\prime}$, and (3) is void. If $x^{\prime} \notin V\left(G_{i}\right)$, then it follows from the 2-connectedness of $G$ that it can be connected to some $y \in V\left(G_{i}\right), y \neq x$, by a simple path in $G-G_{i}$. Obviously, $x$ precedes $y$ on the list LINK, so in this case we can execute (2) and (3) without adding any edge oriented backward with respect to the revised LINK list, and we can pass to the next step.

Note that in the last part of Step $i$, when we revise the LINK list, we do not add any elements below $x$. Since all elements preceding $x$ have already been saturated in $G_{i}$, when we come to part (1) of Step $i+1$, we do not have to check any member of LINK before $x$. 
To execute part (2) of Step $i$, we can use depth-first search on $G-G_{i}$, starting at $x$ and stopping when we hit the first vertex $y \in V\left(G_{i}\right)$. We put every edge $e$ of the tree visited during the search in a (last-in, first-out) stack, and remove $e$ if we have to "backtrack" along it. At the end of the search, the edges remaining in our stack will form a simple path $P_{i+1} \subseteq G-G_{i}$ meeting the requirements. However, unless we are lucky, this procedure may take $\Omega\left(\left|E\left(G-G_{i}\right)\right|\right)$ time, and, summing over all $0 \leq i \leq k$, the total running time of our algorithm can be as large as $\Omega(\mathrm{km})$.

\subsection{The Planar Case}

If $G$ is a plane graph, then the above algorithm can be implemented in $O(m)$ time. Suppose that, for every vertex $v$, we are given the clockwise circular order of the edges incident to $v$. We follow the same scheme as in the general case. To execute part (2) of Step $i$, we use the following method that can be called "left-first search."

Whenever we orient a new edge $f$ toward one of its endpoints $z$, then we set $\operatorname{IN}[z]=f$. Furthermore, let $\mathrm{IN}[s]=s t$.

Assume that we have already finished part (1) of Step $i$, i.e., we have found the first unsaturated vertex $x \in V\left(G_{i}\right)$ on the list LINK. Starting from $x_{0}=x$, we construct the path $P_{i+1}=x_{0} x_{1} x_{2} \ldots$ as follows. For every $j \geq 0$, let $x_{j} x_{j+1}$ be the first unoriented edge incident to $x_{j}$, which follows $\mathrm{IN}\left[x_{j}\right]$ in the clockwise order. Orient $x_{j} x_{j+1}$ from $x_{j}$ toward $x_{j+1}$. If $x_{j+1} \in V\left(G_{i}\right)$, then it is the last point of $P_{i+1}$, and part (2) of Step $i$ has been completed.

To prove that this construction is correct, it is enough to check that $x_{j+1} \neq x_{h}$ for any $h \leq j$. However, this is true, otherwise $x_{h} x_{h+1} \cdots x_{j+1}$ would bound a face of $G$, and $x_{j} x_{j+1}=x_{h} x_{j}$ would precede $x_{h} x_{h+1}$ in the cyclic order of edges around $x_{h}$, contradicting the choice of $x_{h+1}$.

It remains to show that our algorithm can be implemented in linear time. To this end, whenever we orient an edge $\overrightarrow{z z^{\prime}}$, then we introduce a pointer NEXT[ $\left.z\right]$ pointing to the edge that follows immediately after $z z^{\prime}$ in the clockwise order of edges incident to $z$.

Let $x=x_{0} \in V\left(G_{i}\right)$ be the first unsaturated vertex on the list LINK at the beginning of Step $i$ of the algorithm. According to te above rule, next we have to find the first unoriented edge $x_{0} x_{1}$ which comes after $\operatorname{IN}\left[x_{0}\right]$ in the clockwise order of edges incident to $x_{0}$. However, this can be accomplished in constant time, because $x_{0} x_{1}=\operatorname{NEXT}\left[x_{0}\right]$. To prove this, it is enough to notice that the edges oriented toward $x_{0}$ at the beginning of Step $i$ form a single block in the clockwise order of edges incident to $x_{0}$, whose last element is the edge along which $x_{0}$ has been visited for the first time.

Note that the same algorithm can be used to find a Whitney decomposition (and a bipolar orientation) of any cellular graph.

Definition 2.1. A graph $G$ that can be embedded in an oriented 2-manifold $\Sigma$ is called cellular, if it divides $\Sigma$ into connected components so that each of them is topologically equivalent to a disk. 


\section{Bipartite Plane Graphs}

In this section we prove Theorem 1.5. As we have pointed out in the Introduction, we can assume that $G$ is a quadrilateralization (see Definition 1.6), whose vertices are colored black and white. Let $G_{\mathrm{b}}$ and $G_{\mathrm{w}}$ be the graph of black diagonals and the graph of white diagonals of $G$, respectively (see Lemma 1.9). Furthermore, let $s_{\mathrm{b}}, s_{\mathrm{w}}, t_{\mathrm{b}}, t_{\mathrm{w}}$ denote the vertices of the outer face of $G$, listed in clockwise order $\left(s_{\mathrm{b}}, t_{\mathrm{b}} \in V\left(G_{\mathrm{b}}\right) ; s_{\mathrm{w}}, t_{\mathrm{w}} \in V\left(G_{\mathrm{w}}\right)\right)$.

By Corollary $1.10, G_{\mathrm{b}}$ is 2-connected, so we can use the algorithm described in Section 2.2 to find an $s_{\mathrm{b}} t_{\mathrm{b}}$-ordering (bipolar orientation) of $G_{\mathrm{b}}$. That is, in linear time we can number the black vertices $b_{1}=s_{\mathrm{b}}, b_{2}, b_{3}, \ldots, b_{p}=t_{\mathrm{b}}$ so that, orienting every edge of $G_{\mathrm{b}}$ toward its endpoint of larger index, we obtain an $\overrightarrow{s_{\mathrm{b}} t_{\mathrm{b}}}$-bipolar orientation $\vec{G}_{b}$.

$\vec{G}_{\mathrm{b}}$ induces a dual orientation $\vec{G}_{\mathrm{w}}$ on $G_{\mathrm{w}}$. By Theorem 1.4 , reversing the orientation of the edge $\overrightarrow{t_{\mathrm{w}} s_{\mathrm{w}}} \in \vec{G}_{\mathrm{w}}$, we obtain an $\overrightarrow{s_{\mathrm{w}} t_{\mathrm{w}}}$-bipolar orientation $\vec{G}_{\mathrm{w}}^{-}$. Using topological sorting, we can easily find a numbering of the white vertices $w_{1}=s_{\mathrm{w}}, w_{2}, w_{3}, \ldots, w_{q}=t_{\mathrm{w}}$ such that every edge of $\vec{G}_{\mathrm{w}}^{-}$is oriented toward its endpoint of larger index $(p+q=|V(G)|)$.

For any black point $b_{i}(1 \leq i \leq p)$, let $V_{i}$ be a vertical segment in the plane, whose endpoints are $\left(i, \min _{b_{i}, w_{i} \in G} j\right)$ and $\left(i, \max _{b_{i} w_{j} \in G} j\right)$. Similarly, to any white vertex $w_{j}(1 \leq j \leq q)$, we assign a horizontal segment $H_{j}$, whose endpoints are $\left(\min _{b_{i} w_{j} \in G} i, j\right)$ and $\left(\max _{b_{i} w_{j} \in G} i, j\right)$. We claim that this collection of segments meets the requirements of Theorem 1.5.

It is clear by the definition that all segments are contained in the rectangle enclosed by $V_{1}, H_{1}, V_{p}, H_{q}$, and that each of these four segments is in contact with exactly those segments which correspond to its neighbors.

Let us now fix a black point $b_{k}, 1<k<p$, and let $B_{1}=\left\{b_{i} \mid i<k\right\}, B_{2}=\left\{b_{i} \mid i>\right.$ $k\}$. Clearly, the edges connecting $B_{1}$ to $B_{2} \cup\left\{b_{k}\right\}$ form a cocycle $E_{1}$ in $\vec{G}_{\mathrm{b}}$, and the edges connecting $B_{1} \cup\left\{b_{k}\right\}$ to $B_{2}$ form another cocycle $E_{2}$. Since all cocycles of $\vec{G}_{\mathrm{b}}$ are minimal (by Corollary 1.3), the edges of $\vec{G}_{\mathrm{w}}$ intersecting some element of $E_{1}$ $\left(E_{2}\right)$ form a (minimal) oriented cycle $C_{1}\left(C_{2}\right)$ passing through $\overrightarrow{t_{\mathrm{w}} s_{\mathrm{w}}}$. Deleting the edge $\overrightarrow{t_{\mathrm{w}} s_{\mathrm{w}}}$ from $C_{1}$ and $C_{2}$, we obtain two simple oriented paths $P_{1}$ and $P_{2}$, respectively, connecting $s_{\mathrm{w}}$ to $t_{\mathrm{w}}$ in $\vec{G}_{\mathrm{w}}^{-}$. It is easy to see that $b_{k}$ is the only black vertex enclosed by $P_{1}$ and $P_{2}$. Indeed, if there were another vertex $b_{i}$ ( $i<k$, say) with this property, then all vertices along an oriented path connecting $b_{1}=s_{\mathrm{b}}$ to $b_{i}$ in $\vec{G}_{\mathrm{b}}$ would belong to $B_{1}$, hence this path could intersect neither $P_{1}$ nor $P_{2}$, contradiction. On the other hand, since $P_{1}$ and $P_{2}$ are not identical, they must enclose at least one black vertex.

Thus, starting from $s_{\mathrm{w}}, P_{1}$ and $P_{2}$ are identical up to a point $s_{\mathrm{w}}^{\prime}$. Then they split up, and meet again at some point $t_{\mathrm{w}}^{\prime}$, from which they run together to their common endpoint $t_{\mathrm{w}}$. Let $P_{1}^{\prime}$ and $P_{2}^{\prime}$ denote the parts of $P_{1}$ and $P_{2}$, respectively, connecting $s_{\mathrm{w}}^{\prime}$ to $t_{\mathrm{w}}^{\prime}$. Since all edges of $\vec{G}_{\mathrm{b}}$ intersecting some edge of $P_{1}^{\prime}\left(P_{2}^{\prime}\right)$ must end (start) at $b_{k}$, we obtain that all vertices of $P_{1}^{\prime} \cup P_{2}^{\prime}$ are adjacent to $b_{k}$ in $G$. Moreover, $b_{k}$ does not have any other neighbor not belonging to $P_{1}^{\prime} \cup P_{2}^{\prime}$.

Let $W_{1}\left(W_{2}\right)$ denote the set of white points, all of whose black neighbors are in $B_{1}\left(B_{2}\right)$. If a white point $w$ does not belong to $W_{1} \cup W_{2}$, then it must be a vertex of 
$P_{1}$ or $P_{2}$. Indeed, if $w \notin W_{1} \cup W_{2}$, then it has two consecutive neighbors $b$ and $b^{\prime}$ such that, say, $b \in B_{1}$ and $b^{\prime} \notin B_{1}$. However, then $\overrightarrow{b b^{\prime}}$ belongs to the cocycle $E_{1}$ in $\vec{G}_{\mathrm{b}}$, so the edge of $\vec{G}_{\mathrm{w}}$ crossing $\overrightarrow{b b^{\prime}}$ belongs to $P_{1}$, and one of its endpoints is $w$.

Let $w_{j}(1<j<q)$ be a white vertex, and let $H_{j}$ be the corresponding horizontal segment.

Case 1: $b_{k} w_{j} \notin G$. Then $w_{j} \in W_{1} \cup W_{2}$ or $w_{j}$ is an internal vertex of $P_{1} \cap P_{2}$.

If $w_{j}$ belongs to (say) $W_{1}$, then $\max _{b_{i} w_{j} \in G} i<k$. So $H_{j}$ is to the left of $V_{k}$, and $H_{j} \cap V_{k}=\varnothing$.

Suppose next that $w_{j}$ belongs to (say) the portion of $P_{1} \cap P_{2}$ lying strictly between $s_{\mathrm{w}}$ and $s_{\mathrm{w}}^{\prime}$. Then $j$ is smaller than the index of any white neighbor of $b_{k}$, because all of these neighbors belong to $P_{1}^{\prime} \cup P_{2}^{\prime}$ and can be reached from $w_{j}$ by an oriented path in $\vec{G}_{\mathrm{w}}^{-}$(along $P_{1}$ or $P_{2}$ ). Thus, $H_{j}$ is below $V_{k}$, and $H_{j} \cap V_{k}=\varnothing$.

Case 2: $b_{k} w_{j} \in G$. Then $w_{j}$ belongs to $P_{1}^{\prime} \cup P_{2}^{\prime}$.

If $w_{j}=s_{\mathrm{w}}$ (or $t_{\mathrm{w}}$ ), then $w_{j}$ has the smallest (largest) index among all white neighbors of $b_{k}$, so the lower (upper) endpoint of $V_{k}$ lies on $H_{j}$. Moreover, $V_{k}$ has to touch $H_{j}$ at one of its interior points, because $w_{j}$ must be adjacent to at least one black vertex whose index is smaller than $k$ and to another one whose index is larger than $k$.

Suppose next that $w_{j}$ is an internal point of (say) $P_{1}^{\prime}$. Then the right endpoint of $H_{j}$ is an interior point of $V_{k}$.

This shows that the vertical and horizontal segments assigned to the vertices of $G$ satisfy the conditions of Theorem 1.5. We have also proved that the only pairs of segments that share an endpoint are $\left\{V_{1}, H_{1}\right\},\left\{H_{1}, V_{p}\right\},\left\{V_{p}, H_{q}\right\}$, and $\left\{H_{q}, V_{1}\right\}$. Consequently, the segments $V_{i}$ and $H_{j}(1 \leq i \leq p, 1 \leq j \leq q)$ determine a tiling of the rectangle bounded by $V_{1}, H_{1}, V_{p}, H_{q}$ with smaller rectangles. In a forthcoming paper [FMP], we prove Theorem 1.5 by induction.

\section{Partition into Trees}

The aim of this section is to show that the following theorem of Ringel [R1] and Petrovič [P1] can be easily deduced from the above results.

Corollary 4.1. Let $G$ be a quadrilateralization, and let $s_{\mathrm{b}}, s_{\mathrm{w}}, t_{\mathrm{b}}, t_{\mathrm{w}}$ denote the vertices of the outer face of $G$, listed in clockwise order. Then the edge set of $G$ can be partitioned into two parts, forming a spanning tree of $G-s_{\mathrm{b}}$ and $G-t_{\mathrm{b}}$, respectively.

Consider the $\overrightarrow{s_{\mathrm{b}} t_{\mathrm{b}}}$-bipolar orientation of $G_{\mathrm{b}}$ and the $\overrightarrow{s_{\mathrm{w}} t_{\mathrm{w}}}$-bipolar orientation of $G_{\mathrm{w}}$ constructed in the previous section.

Lemma 4.2. An ordering $v_{1}=s_{\mathrm{b}}, v_{2}, v_{3}, \ldots, v_{n}=t_{\mathrm{b}}$ of the vertex set of $G$ exists, which is compatible with the above $\overrightarrow{s_{\mathrm{b}} t_{\mathrm{b}}}$ - and $\overrightarrow{s_{\mathrm{w}} t_{\mathrm{w}}}$-bipolar orientations and satisfies the condition that every $v_{i}(1<i<n)$ is adjacent to at least one larger and one smaller element. 
Proof. As in the previous section, assign to every vertex $v \in V(G)$ a vertical or horizontal segment with endpoints $\left(x_{1}(v), y_{1}(v)\right)$ and $\left(x_{2}(v), y_{2}(v)\right)$, where $x_{1}(v) \leq$ $x_{2}(v), y_{1}(v) \leq y_{2}(v)$. For any pair of adjacent vertices $v, v^{\prime} \in V(G)$ different from $s_{\mathrm{b}}$ and $t_{\mathrm{b}}$, let $v \leq v^{\prime}$ if and only if $x_{2}(v) \leq x_{1}\left(v^{\prime}\right)$ or $y_{2}(v) \leq y_{1}\left(v^{\prime}\right)$. Furthermore, let $s_{\mathrm{b}}$ be smaller and let $t_{\mathrm{b}}$ be larger than any other element of $V(G)$. It is not hard to check that this relation defines a partial order on $V(G)$ compatible with the partial orders on $V\left(G_{\mathrm{b}}\right)$ and $V\left(G_{\mathrm{w}}\right)$ induced by the corresponding bipolar orientations. Therefore, these three relations have a common extension into a total order of $V(G)$. (It can also be shown that this total order is uniquely determined.)

Now we can finish the proof of Corollary 4.1 as follows. For any black vertex $v_{i} \in V\left(G_{\mathrm{b}}\right)$, let $S_{i}$ denote the vertical segment whose endpoints are $\left(i, \min _{v_{t}, v_{j} \in G} j\right)$ and $\left(i, \max _{v_{i} v_{j} \in G} j\right)$. For any white vertex $v_{j} \in V\left(G_{\mathrm{w}}\right)$, let $S_{j}$ denote the horizontal segment with endpoints $\left(\min _{v_{i} v_{j} \in G} i, j\right)$ and $\left(\max _{v_{i} v_{j} \in G} i, j\right)$. Clearly, two such segments touch each other if and only if the corresponding vertices are adjacent. Moreover, by Lemma 4.2 , every segment $S_{i}(1<i<n)$ will cross the line $y=x$.

Color every edge $v_{i} v_{j} \in G$ by red or green according to whether the point of incidence of $S_{i}$ and $S_{j}$ lies above or below the line $y=x$. Then the red and green edges form a spanning tree of $G-v_{1}$ and $G-v_{n}$, respectively. This completes the proof of Corollary 4.1 .

Assume that there is an enumeration $v_{1}, v_{2}, \ldots, v_{n}$ of the vertices of a graph $G$ and a coloring of its edges with $c$ colors such that each color class can be "drawn on a page," that is, there are no two edges of the same color, $v_{h} v_{j}$ and $v_{i} v_{k}$, with $h<i<j<k$. The smallest number $c$ for which such a representation exists is called the page number of $G$. Notice that the total order of $V(G)$ described in Lemma 4.2 and the red-green coloring defined above also yield the following result.

Corollary 4.3. The page number of any quadrilateralization $G$ is at most two. Moreover, the edges of $G$ can always be drawn on two pages so that each page contains a tree.

\section{References}

[DLR] G. DiBattista, W. P. Liu, and I. Rival: Bipartite graphs, drawings and planarity, Inform. Process. Lett. 36 (1990), 317-322.

[E] J. Ebert: st-ordering of the vertices of biconnected graphs, Computing 30 (1983), 19-33.

[EET] G. Ehrlich, S. Even, and R. E. Tarjan: Intersection graphs of curves on the plane, J. Combin. Theory Ser. B 21 (1976), 8-20.

[ET] S. Even and R. E. Tarjan: Computing an st-numbering, Theoret. Comput. Sci. 2 (1976), 339-344.

[FMP] H. de Fraysseix, P. O. de Mendez, and J. Pach: Representation of planar graphs by segments, in: Intuitive Geometry (K. Böröczky and G. Fejes Tóth, eds.), Colloquia Mathematica Societatis J. Bolyai, North-Holland, Amsterdam, to appear.

[FMR] H. de Fraysseix, P. O. de Mendez, and P. Rosenstiehl: Bipolar orientations revisited, Discrete Math., to appear.

[FPP] H. De Fraysseix, J. Pach, and R. Pollack: How to draw a planar graph on a grid, Combinatorica 10 (1990), 41-51. 
[FRU] S. Földes, I. Rival, and J. Urrutia: Light sources, obstructions and spherical orders, Discrete Math. 102 (1992), 13-23.

[HNZ] I. B.-A. Hartman, I. Newman, and R. Ziv: On grid intersection graphs, Discrete Math. 87 (1991), 41-52.

[LEC] A. Lempel, S. Even, and I. Cederbaum: An algorithm for planarity testing of graphs, in: Theory of Graphs (Internat. Symp., Rome, July 1966, P. Rosenstiehl, ed.), Gordon and Breach, New York, 1967, pp. 215-232.

[OW] R. H. J. M. Otten and J. G. van Wijk: Graph representations in interactive layout design, Proc. IEEE Internat. Symp. on Circuits and Systems, 1978, pp. 914-918.

[P1] V. Petrovič: Decomposition of some planar graphs into trees, Proc. Internat. Conf. on Combinatorics, Keszthely, 1993, p. 48.

[P2] C. R. Platt: Planar lattices and planar graphs, J. Combin. Theory Ser. B 21 (1976), 30-39.

[R1] G. Ringel: Two trees in maximal planar bipartite graphs, J. Graph Theory 17 (1993), 755-758.

[R2] I. Rival: Graphical data structures for ordered sets, in: Algorithms and Order (I. Rival, ed.), NATO ASI Series C, Vol. 255, Kluwer, Dordrecht, 1989.

[R3] P. Rosenstiehl: Embedding in the plane with orientation constraints: the angle graph, Ann. N.Y. Acad. Sci. 1983, pp. 340-346.

[RT] P. Rosentiehl and R. E. Tarjan: Rectilinear planar layouts and bipolar orientations of planar graphs, Discrete Comput. Geom. 1 (1986), 343-353.

[T1] R. Tamassia: A dynamic data structure for planar graph embedding, in: Automata, Languages and Programming (T. Lepistö and A. Salomaa, eds.), Lecture Notes in Computer Science, Vol. 317, Springer-Verlag, Berlin, 1988, pp. 576-590.

[TT1] R. Tamassia and I. G. Tollis: A unified approach to visibility representations of planar graphs, Discrete Comput. Geom. 1 (1986), 321-341.

[TT2] R. Tamassia and I. G. Tollis: Centipede graphs and visibility on a cylinder, in: GraphTheoretic Concepts in Computer Science (G. Tinhofer and G. Schmidt, eds.), Lecture Notes in Computer Science, Vol. 246, Springer-Verlag, Berlin, 1987, pp. 252-263.

[TT3] R. Tamassia and I. G. Tollis: Tessellation representations of planar graphs, Proc. 27th Allerton Conf. on Communications, Control, and Computing, 1989, pp. 48-57.

[T2] R. E. Tarjan: Two streamlined depth-first search algorithms, Fund. Inform. 9 (1986), 85-94.

[W] H. Whitney: On the classification of graphs, Amer. J. Math. 55 (1933), 236-244.

Received January 25, 1994, and in revised form September 6, 1994. 